\title{
Does toluidine blue detect more oral cancer?
}

\author{
Abstracted from
}

Su WW, Yen AM, Chiu SY, Chen TH.

A community-based RCT for oral cancer screening with toluidine blue. J Dent Res 2010; 89: 933-937.

Address for correspondence: T.H.-H. Chen, Centre of Biostatistics Consultation,

College of Public Health, National Taiwan University, Taiwan. E-mail: chenlin@ntu.edu.tw

\section{Question: Does the use of toluidine blue as an adjunctive tool for visual screening of the mouth result in the increased detection of oral premalignant lesions (OPMLs)?}

\section{Design A randomised controlled trial.}

Intervention 28,167 individuals aged 15 years or older who were invited participate in a screening programme, 17,890 who lacked oral habits such as cigarette smoking or chewing betel quid were excluded leaving 10,277 eligible individuals. Of these, 2,302 refused to participate leaving a sample of 7,975 for randomisation. The test group gargled with toluidine blue solution while the control group was given a placebo dye of the same colour. Each participant was visually examined by one of six dentists with additional training. The presence of any visible lesion in the oral cavity (e.g. abnormal mucosal lesions related to OPMLs and other suspected lesions such as lichen planus, oral ulcer, hyperkeratosis, candidiasis, etc) was recorded as screenpositive. The screen-positive participants to a specialist were referred to a specialist for a definite clinical diagnosis within 10 to 14 days, to reduce false-positivity, and biopsies were arranged if oral lesions were present. The occurrence of oral cancer, survival status, and causes of death of the studied participants for the entire cohort was obtained from the National Cancer Registry and the National Household Registry until the end of 2004.

Outcome measure The number of oral lesions, premalignant or not.

\begin{tabular}{|c|c|c|c|c|c|}
\hline & \multicolumn{2}{|l|}{ Test } & \multicolumn{2}{|c|}{ Control } & \multirow{2}{*}{$\begin{array}{l}\text { Rate Ratio } \\
\text { ( } 95 \% \text { confidence } \\
\text { interval) }\end{array}$} \\
\hline & $n$ & $\%$ & $\mathrm{n}$ & $\%$ & \\
\hline Negative & 55 & 1.3 & 59 & 1.5 & $0.82(0.55-1.24)$ \\
\hline Non-OPMLs & 78 & 1.9 & 64 & 1.6 & $1.15(0.79-1.68)$ \\
\hline $\begin{array}{l}\text { All OPMLs and } \\
\text { malignant lesions }\end{array}$ & 187 & 4.6 & 170 & 4.4 & $1.05(0.74-1.41)$ \\
\hline Oral submucous fibrosis & 41 & 1.0 & 22 & 0.6 & $1.79(1.06-3.01)$ \\
\hline $\begin{array}{l}\text { Homogeneous } \\
\text { leukoplakia }\end{array}$ & 125 & 3.1 & 131 & 3.4 & $0.79(0.57-1.09)$ \\
\hline $\begin{array}{l}\text { Non-homogeneous } \\
\text { leukoplakia }\end{array}$ & 13 & 0.3 & 11 & 0.3 & $1.08(0.48-2.46)$ \\
\hline Erythroplakia & 7 & 0.2 & 6 & 0.2 & $1.07(0.36-3.22)$ \\
\hline
\end{tabular}

Results In the test group $389(9.5 \%)$ individuals had a positive screen compared with 322 (8.3\%) in the control group. Two oral cancers were identified out of those with a positive screen presenting $(n=320)$ for the referral examination from the test group with three oral cancers out of the 293 in those who complied in the control group. There were three oral cancers ascertained by the end of the follow-up among the screen-negatives in each arm (test and control) by the end of the followup period. The numbers of OPMLs in each arm of the study are shown in table 1.

Conclusions We demonstrated that using toluidine blue as an adjunctive tool for visual screening can detect significantly more oral submucous fibrosis and slightly more leukoplakia among high-risk individuals with habits of cigarette smoking or betel quid chewing as compared with visual screening alone.

\section{Commentary}

This paper presents an interesting trial of a possible screening programme for oral cancer in Taiwan. Oral cancers are a significant group of diseases worldwide being the sixth most common cancer and accounting for about $4 \%$ of cancers, and in some parts of the world the incidence is increasing. ${ }^{1-2}$ There is strong evidence that tobacco use, alcohol consumption and betel quid chewing are the main risk factors in the aetiology of intraoral cancer. ${ }^{3-4}$ Despite significant technical advances in the treatment of oral cancer, survival following a diagnosis of oral cavity or oropharyngeal cancer remains poor with 5-year survival around 50\% overall, with only limited improvement in the past 3 decades. ${ }^{2}$ The stage of the disease has a bearing on the outcome of the disease and yet oropharyngeal cancers have relatively 'silent' symptoms which may not be present during the early stages of the disease, which is a possible explanation for the fact that stage of disease at diagnosis has not altered in the past 40 years despite public education. ${ }^{5}$

For these reasons there is an interest in the early identification of oral cancer cases yet the case for formal screening programmes does not meet the criteria established by the UK screening committee (www.screening.nhs.uk/criteria). The UK National Screening Committee reviewed their policy on oral cancer screening in June of this year and reaffirmed their previous position that screening should not be offered.

The screening programme presented in the current paper identifies around $4.5 \%$ of OPMLs. However the differences between the test and control dyes are very small for all the lesions apart from those patients with oral submucous fibrosis. The fact that there is 
little difference between the two dyes raises as many questions as it answers.

The traditional way to measure the utility of a screening test is by determining the sensitivity and specificity of the tests. However, sensitivity and specificity data are not presented. Indeed, it is not apparent from the paper whether there were any OPMLs present in those patients who screened negative (i.e. the false negatives). Even though no information is provided on the number of false negative OPMLs, because of the linkage of the screened cohort with the cancer registry data there is information on the number of oral cancers in this cohort of patients in both arms of the study. Consequently by using this data it is possible to estimate the sensitivity, specificity, positive and negative predictive values and likelihood values for the test and control dyes in the identification of oral cancer (Table $2 \& 3$ ).

\begin{tabular}{|l|l|l|l|}
\hline \multicolumn{4}{|c|}{ Table 2 Sensitivity and Specificity for Toluidine Blue } \\
\hline & Oral Cancer & No Cancer & totals \\
\hline Positive screen & 2 & 387 & 389 \\
\hline Negative screen & 3 & 3688 & 3691 \\
\hline totals & 5 & 4075 & 4080 \\
\hline
\end{tabular}

Sensitivity 0.400 Positive predictive value 0.005 Likelihood ratio 4.2

Specificity 0.905 Negative predictive value 0.999

Table 3 Sensitivity and Specificity for placebo dye

\begin{tabular}{l|l|l|l} 
& Oral Cancer & No Cancer & totals \\
\hline Positive screen & 3 & 319 & 322 \\
\hline Negative screen & 3 & 3570 & 3573 \\
\hline totals & 6 & 3889 & 3895
\end{tabular}

Sensitivity 0.500 Positive predictive value 0.009 Likelihood ratio 6.1

Specificity 0.918 Negative predictive value 0.999

These positive predictive values (PPVs; proportion of people with the symptom, sign, or test result who develop cancer) for the use of dyes to detect oral cancer are pretty low - less than 1\% . A recent review $^{6}$ of positive predictive values for symptoms, signs and nondiagnostic test results for cancer or suspicion of cancer in general medical care suggest that values for PPVs for cancer should be $\geq 5 \%$.

It should be borne in mind that the aim of this study was to identify OPMLs and not oral cancer per se and it may well be that the PPVs for identifying OPMLs is higher that $5 \%$ indicating that the test may have some utility. While screening may identify OPMLs that require further management, screening may also have potential negative effects. Screening whether on an individual or population perspective is far from perfect, and there are four potential outcomes from any test;

- true positive (correctly identified as positive),

- true negative (correctly identified as negative

- false positive (incorrectly identified as positive)

- false negative (incorrectly identified as negative)

False negatives can provide a false sense of security and perpetuate bad habits or delay final diagnosis.

False positives can cause stress and anxiety and lead to unnecessary additional appointments, tests and investigations. From the data presented we are unable to calculate the false negative proportions for OPMLs but if we consider those in this study who screen positive but are subsequently found to be have no lesions or a nonOPMLs to be negative, then the proportion of false positives was about $42 \%$ in each group.

Based on the evidence presented in the paper I find it difficult to agree with the conclusions that toluidine blue dye is more effective than visual screening alone. What I find more interesting is that the placebo dye is almost as effective. Overall this study, which has been published since the most recent update of the Cochrane review on oral cancer screening and the clinical recommendations from the ADA on oral cancer screening, does little to change my view that formal screening programmes for oral cancer are not a public health intervention that I would support.

\section{Practice points}

- The currently available evidence does not provide support for population screening programmes for oral cancer.

\section{Derek Richards}

Centre for Evidence-based Dentistry, Oxford.

1. Conway DI, Stockton DL, Warnakulasuriya KA, Ogden G, Macpherson LM. Incidence of oral and oropharyngeal cancer in United Kingdom (1990-1999) - recent trends and regional variation. Oral Oncol 2006; 42: 586-592. Epub 2006 Feb 15.

2. Warnakulasuriya $S$. Global epidemiology of oral and oropharyngeal cancer. Oral Oncol 2009; 45: 309-316. Epub 2008 Sep 18.

3. La Vecchia C, Tavani A, Franceschi S, Levi F, Corrao G, Negri E. Epidemiology and prevention of oral cancer. Oral Oncol 1997; 33: 302-312.

4. International Agency on Research on Cancer. Betel-quid and areca-nut chewing and some areca-nut related nitrosamines. IARC monographs on the evaluation of carcinogenic risks to humans., Vol. 85. IARC; 2004.

5. McGurk M, Chan C, Jones J, O'regan E, Sherriff M. Delay in diagnosis and its effect on outcome in head and neck cancer. Br J Oral Maxillofac Surg 2005; 43: 281-284.

6. Shapley M, Mansell G, Jordan JL, Jordan KP. Positive predictive values of $\geq 5 \%$ in primary care for cancer: systematic review. Br J Gen Pract 2010; 60: e366-377.

Evidence-Based Dentistry (2010) 11, 104-105. doi:10.1038/sj.ebd.6400752 